Report

\title{
Inter-laboratory Study for Validation of a Japanese Official Analytical Method for Determination of Patulin in Apple Juice
}

(Received February 14, 2005)

\author{
Yoshiko Sugita-Konsini*1, ${ }^{\dagger}$, Toshitugu Tanaka*2, Yoshitugu Sugiura*2, \\ Setsuko Tabata*3, Masahiro NAKajima*4, Hiroyuki Sakurai*5, Yoko Nakaie*6, \\ Koichi Sato*7, Yusuke Kitani*7, Kazuhiro Fujita*8, Sachiko Hayashi*8, Tayoshi Iizuka*9, \\ Yoshinori Hirakawa*9, Naoki Mochizuki*10, Mariko Hoshino*10, Yoshirou Sato*11, \\ Naomi TAKAHASHI ${ }^{* 11}$ and Kosuke TAKATORI ${ }^{* 1}$ \\ (*1 National Institute of Health Sciences: 1-18-1, Kamiyoga, Setagaya-ku, Tokyo 158-8501, Japan; \\ *2 Kobe Institute of Health: 4-6, Minatojima-nakamachi, Chuo-ku, Kobe 650-0046, Japan; \\ *3 Tokyo Metropolitan Institute of Public Health: 3-24-1, Hyakunin-cho, \\ Shinjuku-ku, Tokyo 169-0073, Japan; \\ *4 Nagoya City Public Health, Research Institute: 1-11, Hagiyama, Mizuho-ku, \\ Nagoya 467-8615, Japan; \\ *5 Yokohama Quarantine Station: 107-8, Nagahama, Kanazawa-ku, Yokohama 236-0011, Japan; \\ *6 Kobe Quarantine Station: 1-1, Toyahamacho, Kobe, Hyogo 230-0862, Japan; \\ *7 IAA Center for Food Quality, Labeling and Consumer Services: 2-1, \\ Shintoshin, Chuo-ku, Saitama 330-9731, Japan; \\ *8 Japan Food Research Laboratories, Nagoya Branch: 4-5-13, Otu, \\ Naka-ku, Nagoya 460-0011, Japan; \\ *9 Japan Food Research Laboratories, Japan Inspection Association of Food and \\ Food Industry Environment: 3-7-4, Kyobashi, Chuo-ku, Tokyo 104-0031, Japan; \\ *10 Asahi Breweries, Ltd.: 1-1-21, Midori, Moriya-shi, Ibaraki 302-0106, Japan; \\ *11 Meiji Dairies Corporation: 540, Naruta, Odawara, Kanagawa \\ 250-0862, Japan; ${ }^{\dagger}$ Corresponding author)
}

To validate a modified version of AOAC official method of analysis 995.10 as an official standard in Japan for determination of patulin in apple juice, an inter-laboratory study was performed in 11 laboratories using a non-contaminated sample, 2 naturally contaminated samples and 2 spiked samples of apple juice. For naturally contaminated apple juices, the relative standard deviations for repeatability and reproducibility were $3.2,7.1 \%$ and $10.0,21.7 \%$, respectively. HORRAT values were $0.4,0.9$. The average recovery of patulin from spiked sample was $83.7 \%$. The limit of quantification was calculated as $10 \mu \mathrm{g} / \mathrm{kg}$. From these results, the method was thought to be suitable as an official standard for determination of patulin in apple juice in Japan.

Key words: apple juice; patulin; official method; HPLC; inter-laboratory study; validation

\section{Introduction}

Patulin is a mycotoxin which contaminates fruit juices, especially apple juice. It is mainly produced by Penicillium expansum. The toxicity of patulin was evaluated in 1996 by the Joint FAO/WHO Expert Committee on Food Additives (JECFA) ${ }^{1)}$, which concluded that the provisional maximal tolerable daily intake of patulin is $0.4 \mu \mathrm{g} / \mathrm{kg}$ body weight/day.

The Codex committee has recommended a maximum level of patulin in apple juice of $50 \mu \mathrm{g} / \mathrm{kg}$ and many countries have established a standard for patulin. In Japan, the Ministry of Health, Labour and Welfare established a limit of $50 \mu \mathrm{g} / \mathrm{kg}$ in $2004^{2)}$. To enforce this, an official method for determination of patulin in apple juice should be established.

Several analytical methods for measuring levels of patulin have been developed, based on highperformance liquid chromatography (HPLC) ${ }^{3), 4)}$,

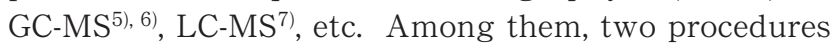
using ethyl acetate for extraction and sodium carbonate for cleaning-up before HPLC have been published, AOAC official methods $995.10^{3)}$ and $2000.02^{4)}$, and are used in many countries to determine the concentration of patulin in apple juice. However less organic solvent and time are required for 995.10 than 2000.02. Therefore, in this study, an HPLC-based analytical procedure modified from method 995.10 was validated as an 
official standard for determination of patulin in apple juice in an inter-laboratory study involving 11 laboratories.

\section{Materials and Methods}

\section{Standard and reagents}

A patulin standard was purchased from Sigma Chemicals Co. (St. Louis, MO, USA). The patulin (5 mg) was weighed into a $50 \mathrm{~mL}$ volumetric flask. The toxin was dissolved in ethyl acetate as a stock solution and stored in a freezer at $-20^{\circ} \mathrm{C}$. One milliliter of this stock solution was evaporated to dryness under a stream of nitrogen gas, and the residue was immediately dissolved in $10 \mathrm{~mL}$ of ethanol $(c a .10 \mu \mathrm{g} / \mathrm{mL})$. The UV absorbance was measured, and the concentration of patulin was calculated. Two more portions of the stock solution of patulin were appropriately diluted with acetonitrile to prepare spiked solutions $(246.7 \mathrm{ng} / \mathrm{mL}$ and $101.2 \mathrm{ng} / \mathrm{mL}$ ) in the laboratory (National Institute of Health Sciences). Organic solvents, such as ethyl acetate and acetonitrile of HPLC grade, water of HPLC grade, and reagents were purchased from Wako Pure Chemical Industries, Ltd. (Osaka, Japan).

\section{Preparation of samples}

Clear and clouded samples of apple juice naturally contaminated with patulin, which were used in the Food Analytical Performance Assessment Scheme (FAPAS) Program carried out in 2003, were purchased from Central Science Laboratories (York, UK). The assigned concentrations of patulin in these samples were $22.5 \mu \mathrm{g} / \mathrm{kg}$ in the clear apple juice and $49.8 \mu \mathrm{g} / \mathrm{kg}$ in the clouded apple juice, according to the FAPAS report. Commercial clear type apple juice in which the patulin content was found to be below the limit of quantification using procedure 995.10, the official AOAC method of analysis ${ }^{3)}$, was selected as a blank.

\section{Fortification procedure}

For recovery determinations, a $1 \mathrm{~mL}$ aliquot of each spiked solution was taken into a 30 to $50 \mathrm{~mL}$ centrifuge tube and evaporated under nitrogen gas to eliminate acetonitrile. Five milliliters of non-contaminated apple juice was immediately added to individual tubes, and mixed well (equivalent to fortification levels of $49.3 \mu \mathrm{g}$ / $\mathrm{kg}$ and $20.2 \mu \mathrm{g} / \mathrm{kg}$ patulin, respectively). One hour later, the toxin was extracted and clean-up was carried out according to the protocol described in this paper.

\section{Protocol used by participants}

Scheme 1 shows the protocol for the analytical procedure (Japanese official method) modified from method 995.10. Briefly, $5 \mathrm{~g}$ of apple juice was placed in a 30 to $50 \mathrm{~mL}$ centrifuge tube, and $10 \mathrm{~mL}$ of ethyl acetate was added. After $1 \mathrm{~min}$ of shaking, the ethyl acetate layer was transferred to a new centrifuge tube. This extraction procedure was repeated three times. These ethyl acetate extracts were combined in the centrifuge tube, 2 $\mathrm{mL}$ of $1.5 \%$ sodium carbonate solution was added, and the tube was shaken vigorously for $10-20 \mathrm{sec}$. The ethyl acetate layer was transferred to a clean tube, and the lower aqueous layer was extracted with $5 \mathrm{~mL}$ of ethyl acetate. Then the ethyl acetate layer was added to the tube and evaporated to dryness under nitrogen gas.

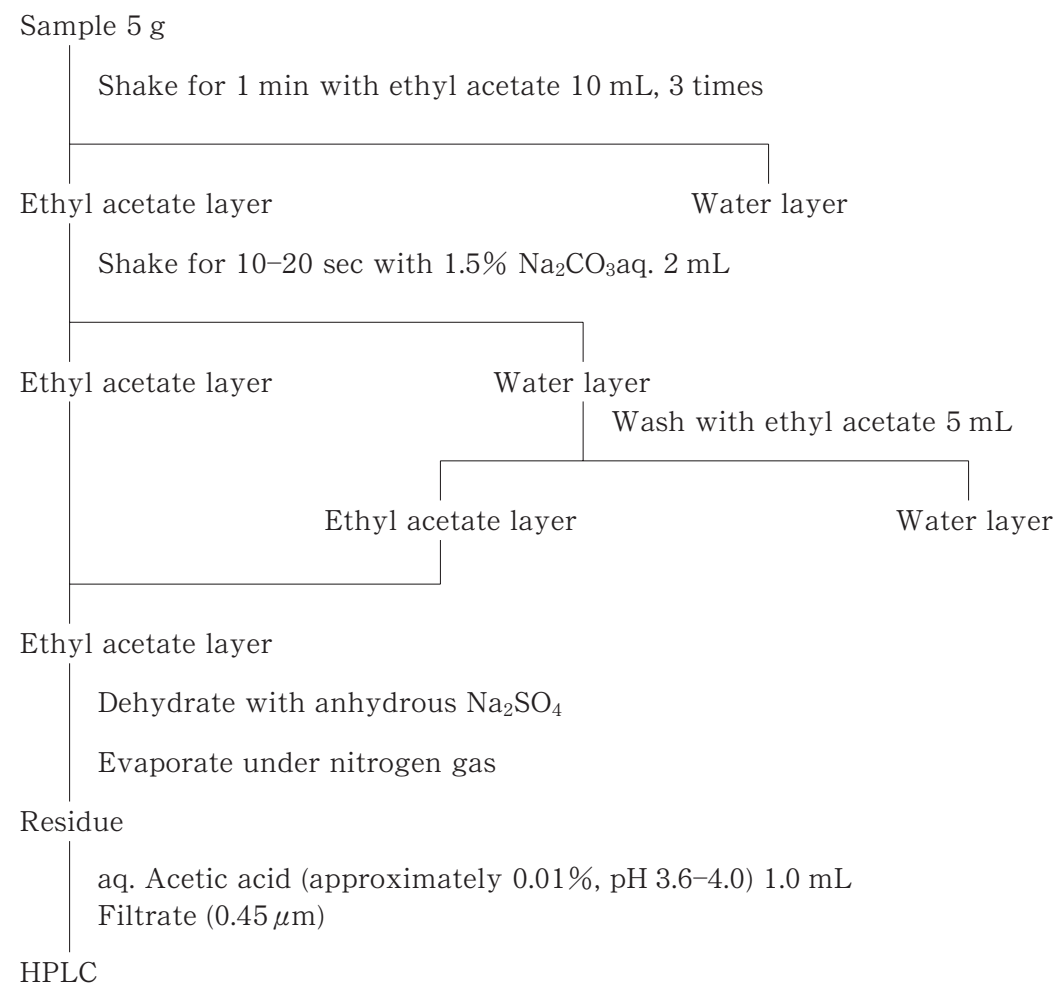

Scheme 1. Protocol of the method for determination of patulin in apple juice validated with an inter-laboratory study 
Table 1. HPLC Conditions

Column: ODS $100-250 \mathrm{~mm} \times 4.6 \mathrm{~mm}$ i.d. $(3-5 \mu \mathrm{m})$

Column temp.: $40^{\circ} \mathrm{C}$

Flow rate: $1.0 \mathrm{~mL} / \mathrm{min}$

Wavelength: $276 \mathrm{~nm}(290 \mathrm{~nm})$

Injected volume: $40 \mu \mathrm{L}$

Mobile phase: Acetonitrile-water (4:96)

The residue was dissolved in $1 \mathrm{~mL}$ of acetic acid (approximately $0.01 \%$, pH 3.6-4.0). After filtration, the sample was subjected to HPLC analysis.

\section{HPLC conditions}

HPLC conditions are listed in Table 1 . The analytical ODS column $(100-250 \mathrm{~mm} \times 4.6 \mathrm{~mm}$ i.d., $3-5 \mu \mathrm{m})$ was kept at $40^{\circ} \mathrm{C}$ and the mobile phase was acetonitrilewater $(4: 96 \mathrm{v} / \mathrm{v})$, delivered at a flow rate of $1.0 \mathrm{~mL} /$ min. Detection was performed with a UV detector at a wavelength of $276 \mathrm{~nm}$.

\section{Inter-laboratory study}

For validation of the official method, an interlaboratory study was carried out using 5 materials (two naturally contaminated apple juices, two spiked apple juices and a blank) according the protocol of the International Union of Pure and Applied Chemistry $(\mathrm{IUPAC})^{8)}$. Eleven laboratories participated in the interlaboratory study, that is, National Institute of Health Sciences, Kobe Institute of Health, Nagoya City Public Health Research Institute, Tokyo Metropolitan Insti- tute of Public Health, Yokohama Quarantine Station, Kobe Quarantine Station, IAA Center for Food Quality, Labeling and Consumer Services, Japan Food Research Laboratories, Nagoya Branch, Japan Inspection Association of Food and Food Industry Environment, Asahi Breweries, Ltd. and Meiji Dairies Corporation.

\section{Statistics}

The raw data sent by the participants were subjected to statistical tests (Cochran, Grubbs and Grubbs paired tests) according to the AOAC guide ${ }^{9)}$. First the suspect and outlying data between blind duplicates (Corhran test) and between laboratory means (Grubbs test) were analyzed. Then, the parameters of precision, which are the inter-laboratory relative standard deviations for repeatability $\left(\mathrm{RSD}_{\mathrm{r}}\right)$ and for reproducibility $\left(\mathrm{RSD}_{\mathrm{R}}\right)$, were deduced.

\section{Results and Discussion}

The procedure used in this study was a modified version of AOAC official method of analysis 995.10 in which tetrahydrofuran was omitted from the mobile phase, because it is known to be corrosive.

Five samples were used in this inter-laboratory study, non-contaminated, clear type naturally contaminated, clouded type naturally contaminated and two concentrations of spiked apple juice. The number of materials satisfied the minimum for a quantitative study using the collaborative study protocol of IUPAC. The results received from the 11 laboratories regarding the concentration of patulin in naturally contaminated apple juice

Table 2. Inter-laboratory Results (Blind Duplicate) for Determination of Patulin in Apple Juice by the Method Modified from the AOAC Official Method 995.10

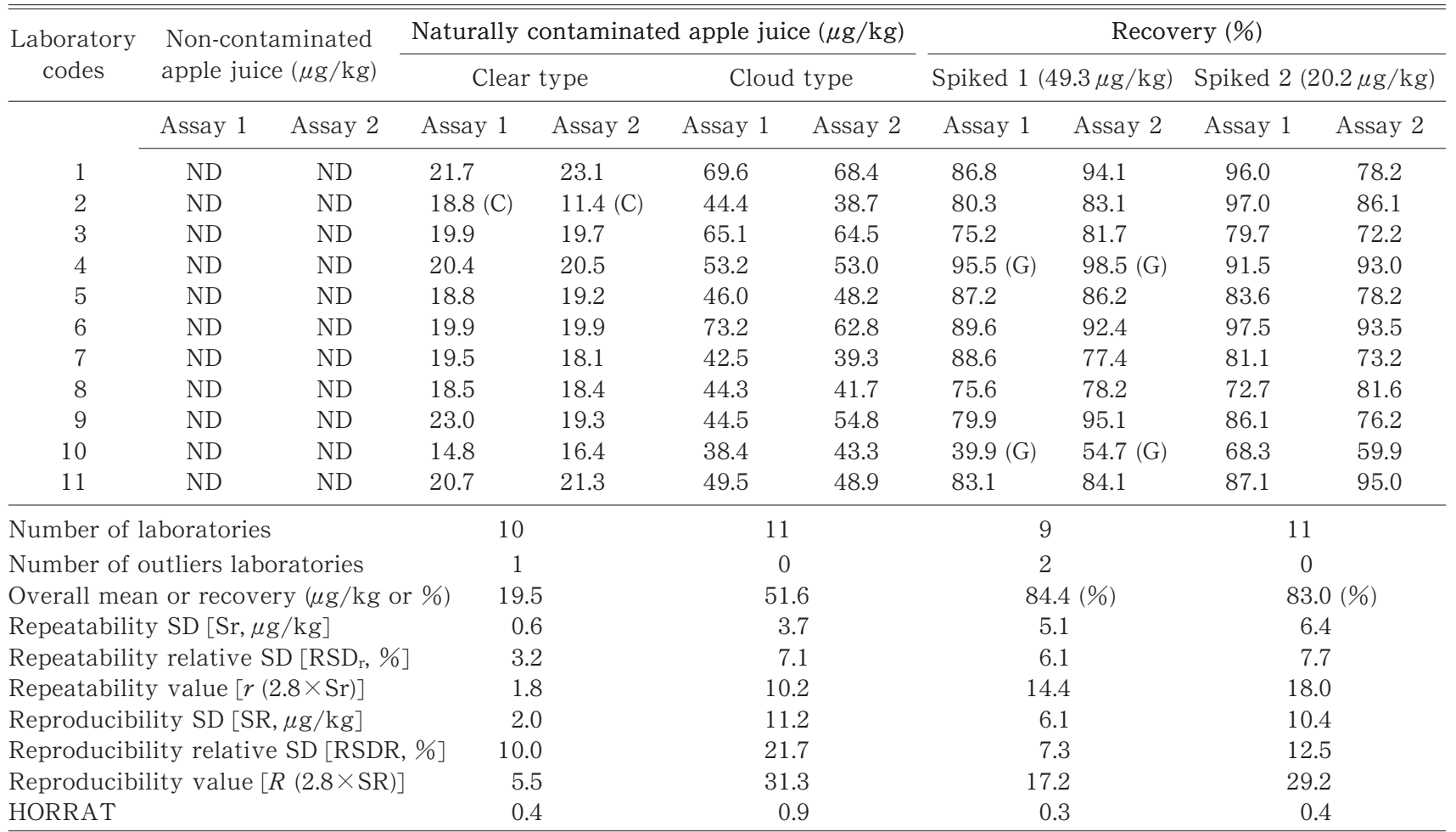

Abbreviations: $\mathrm{C}=$ Cochran outlier, $\mathrm{G}=$ Grubbs outlier, $\mathrm{ND}=$ Not determined 
and the rate of recovery are listed in Table 2.

Performance parameters are shown for 1 case of an outlier in clear naturally contaminated apple juice and for 2 cases of an outlier in spiked juice. For noncontaminated apple juice, no laboratory detected patulin at a concentration above the level of the limit of detection. For the naturally contaminated juices, the $\mathrm{RSD}_{\mathrm{r}}$ was $3.2 \%$ for clear type and $7.1 \%$ for clouded type, calculated without outliers. The $\mathrm{RSD}_{\mathrm{R}}$ was $10.0 \%$ for clear type and $21.7 \%$ for clouded type. The HORRAT value was 0.4 for clear type and 0.9 for clouded type.

Tests were also carried out to determine the recovery from the samples spiked with $49.3 \mu \mathrm{g} / \mathrm{kg}$ or $20.2 \mu \mathrm{g} / \mathrm{kg}$ of patulin. For the two spiked samples, the mean recovery rate was $83.7 \%$, calculated without outliers. For mycotoxins, a method to be recognized as an official method is required to have a recovery in the range of $70-110 \%{ }^{10)}$. Hence, the rate of recovery obtained in the inter-laboratory study was acceptable. The $\mathrm{RSD}_{\mathrm{r}}$ values of the samples spiked with $49.3 \mu \mathrm{g} / \mathrm{kg}$ and 20.2 $\mu \mathrm{g} / \mathrm{kg}$ of patulin were $6.1 \%$ and $7.7 \%$ and the $\mathrm{RSD}_{\mathrm{R}}$ values were $7.3 \%$ and $12.5 \%$, respectively. The HORRAT value was 0.3 for the sample spiked with $49.3 \mu \mathrm{g} /$ $\mathrm{kg}$ of patulin and 0.4 for that spiked with $20.2 \mu \mathrm{g} / \mathrm{kg}$ of patulin, respectively.

In the calibration curve, good linearity with a correlation coefficient of $>0.9997$ was obtained in the range of $50-500 \mathrm{ng} / \mathrm{mL}$ of patulin standard solution. From the $S / N(=3)$ value, the limit of detection was calculated as $3.0 \mu \mathrm{g} / \mathrm{kg}$ and the limit of quantification was calculated as $10.0 \mu \mathrm{g} / \mathrm{kg}$ from the minimum dose obtained from the linear calibration curve. Since the maximum level of patulin allowable in apple juice in Japan is $50 \mu \mathrm{g} / \mathrm{kg}$, this method is effective to $1 / 5$ of the maximum level.

Brause et al. ${ }^{11)}$ performed an international collaborative study using the method 995.10. In their case, the average recorery was $96 \%$, and the $\mathrm{RSD}_{\mathrm{r}}$ and the $\mathrm{RSD}_{\mathrm{R}}$ ranged from 10.9 to $53.8 \%$ and from 15.1 to $68.8 \%$, respectively. In our study, though the average recovery was lower than their results, satisfactory parameters of precision were obtained. Recently, Arranz et al. ${ }^{12)}$ reported an inter-laboratory study using a method that involves extraction with ethyl acetate, followed by a silica gel solid-phase extraction clean-up. The results showed that the method allows determination of patulin at $10 \mu \mathrm{g} / \mathrm{kg}$ with sufficient precision. In future, if the maximum level of patulin in apple-based products intended for infants is set at $25 \mu \mathrm{g} / \mathrm{kg}$ in Japan, as has been suggested, it will be necessary to validate a more precise method. However, as the maximum limit of patulin in apple juice is set at $50 \mu \mathrm{g} / \mathrm{kg}$ at present, the method validated in this study can be adopted as the official standard in Japan.

Tabata et al. ${ }^{6)}$ pointed out that the UV spectrum of the interfering peak close to patulin was very similar to that of patulin when a wavelength of $276 \mathrm{~nm}$ was used for detection. To distinguish patulin from the interfering peak, AOAC recommends using a non end-capped
HPLC column with a carbon load of $16 \%$ in both official methods. Further, our study revealed that the interfering peak was reduced ${ }^{13)}$ when UV detection was performed at $290 \mathrm{~nm}$. Good linearity in the calibration curve was obtained at $290 \mathrm{~nm}$ as well as at $276 \mathrm{~nm}$, though the sensitivity of detection was declined 30\% lower in comparison with that at $276 \mathrm{~nm}$. Therefore, close attention to selecting the HPLC column and a wavelength of $290 \mathrm{~nm}$ are recommended for the official Japanese method.

\section{Acknowledgements}

This study was supported by a grant for Food Hygienic Research, the Japanese Ministry of Health, Labour and Welfare.

\section{References}

1) Joint FAO/WHO Expert committee on Food Additives $44^{\text {th }}$ Session ed. "Toxicological evaluation of certain food additives and contaminants in food., Geneva, 1996, p. 377-402. (ISDN 92-4-166035-X)

2) Notification No. 369 (Nov. 26, 2003) Ministry of Health, Labour and Welfare, Japan.

3) Truckness, N.W. (ed.), Natural toxins, AOAC official methods of analysis, $17^{\text {th }}$ Ed., 2000, Chapter 49, p. 50-53.

4) MacDonald, S. M., Long, M., Gilbert, J., Liquid chromatographic method for determination of patulin in clear and cloudy apple juices and apple puree; collaborative study. J. AOAC Int., 83, 1,387-1, 394 (2000).

5) Rupp, H. S., Turnipseed, S. B., Confirmation of patulin and 5-hydroxymethylfurfural in apple juice by gas chromatography/mass spectrometry. J. AOAC Int., 83, 612-620 (2000).

6) Roach, J. A., White, K. D, Trucksess, M. W., Thomas, F. S., Capillary gas chromatography/mass spectrometry with chemical ionization and negative ion detection for confirmation of identity of patulin in apple juice. J. AOAC Int., 83, 104-112 (2000).

7) Tabata, S., Iida, K., Suzuki, J., Kimura, K., Ibe, A., Saito, K., A quantification and confirmation method of patulin in apple juice by GC/MS. J. Food Hyg. Soc. Japan, 45, 245249 (2004)

8) Horwitz, W., Protocol for the design, conduct and interpretation of methodperformance studies. Pure Appl. Chem., 67, 331-343 (1995).

9) Youden, W., Steiner, E. H. "Statistical manual of the Association of Official Analytical Chemists", Arlington, Association of Official Analytical Chemists, 1975, p. 7283. (ISBN 0-935584-15-3)

10) Gilbert, J., Validation of analytical methods for determining mycotoxins in foodstuffs. Trends in Analytical Chemistry, 21, 468-486 (2002).

11) Brause, A. R., Trucksess, M., Thomas, F. S., Page, W., Determination of patulin in apple juice by liquid chromatography. J. AOAC Int., 79, 451-455 (1996).

12) Arranz, I., Derbyshire, M., Kroeger, K., Midchke, C., Stroka, J., Anklan, E., Liquid chromatographic method for quantitation of patulin at $10 \mathrm{ng} / \mathrm{mL}$ in apple-based products intended for infants: interlaboratory study. J. AOAC Int., 88, 518-525 (2005).

13) Konishi, Y., Test method of patulin. Shokuhin Eisei Kenkyu (Food Sanit. Res.), 54, 11-16 (2004). 